\title{
LETTER TO THE EDITOR \\ Sulfated glycosaminoglycans and low-density lipoprotein receptor mediate the cellular entry of Clostridium novyi alpha-toxin
}

\author{
Cell Research (2021) 31:935-938; https://doi.org/10.1038/s41422-021-00510-z
}

\section{Dear Editor}

Clostridium novyi (C. novyi) is a spore-forming anaerobic bacterium and opportunistic pathogen causing severe infectious diseases in humans and animals including gas gangrene, myositis, necrotic hepatitis, and sepsis. ${ }^{1,2}$ The alpha-toxin (Tcna) is the major virulence factor of $C$. novyi and is produced by all known pathogenic C. novyi types. ${ }^{1}$ Tcna belongs to the large clostridial toxin (LCT) family which also includes Clostridioides difficile toxin A $(\mathrm{Tcd} A)$ and toxin B (TcdB), Paeniclostridium sordellii lethal toxin (TcsL) and hemorrhagic toxin (TcsH), and Clostridium perfringens large cytotoxin (TpeL). Like other LCTs, Tcna binds to the surface of the target cell and enters the cell via receptormediated endocytosis. The enzymatic domain is then delivered into the cytosol and glucosylates small Rho-family GTPases using UDP-N-acetylglucosamine as a co-substrate, leading to cytoskeleton disruption and cell death. ${ }^{3}$ Recently, the cellular receptors of several LCTs, including TpeL, TcdB, TcdA, and TcsL have been identified. $^{4-10}$ Tcna and TcsH are the only two major members of the LCT family with their host receptors remaining unclear.

To identify potential receptors for Tcna, we conducted genomewide CRISPR/Cas9-mediated knockout (KO) screens. HeLa cells stably expressing Cas9 (parental cell line, referred to as the wildtype (WT) thereafter) were transduced with a lentiviral single guide RNA (sgRNA) library (GeCKO v2) and subjected to three rounds of selection with Tcna (Fig. 1a). Next-generation sequencing of sgRNAs from the surviving cells showed that four of the top five enriched genes, including B4GALT7, B3GALT6, EXT1, and $S L C 35 B 2$, encode enzymes involved in heparan sulfate biosynthesis. Several other genes encoding enzymes in the heparan sulfate biosynthesis pathways, such as B3GAT3, EXT2, HS6ST3, and EXTL3 were also greatly enriched ( $>10$-fold) from our screens (Fig. 1b; Supplementary information, Table S1).

To validate the role of heparan sulfate in the cellular binding/

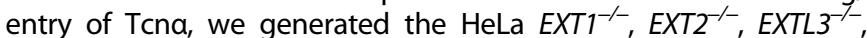
$B 4 \mathrm{GALT7}^{-1-}$, and SLC35B2 ${ }^{-1}$ cells via CRISPR/Cas9-mediated KO. The cytopathic cell rounding experiment (toxin concentration that results in $50 \%$ cell rounding is defined as $C R_{50}$ ) showed that all these $\mathrm{KO}$ cells exhibited increased resistance ( 10 to 30 -fold based on $\mathrm{CR}_{50}$ ) against Tcna compared to the WT cells (Fig. 1c, d). Heparan sulfate is usually anchored to core proteins as heparan sulfate proteoglycans (Supplementary information, Fig. S1) and is abundant on various cell types, thus may serve as universal cell surface attachment factors for Tcna. We labeled Tcna with fluorescent dye Rhodamine and then examined its surface binding to the HeLa cells. Rhodamine-labeled Tcna showed similar toxicity as Tcna

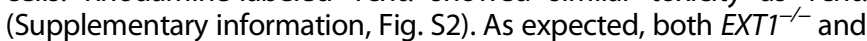
$S L C 35 B 2^{-1-}$ HeLa cells showed drastically reduced surface binding of Tcna when compared with the WT cells (Fig. 1e).
Among the above tested KO cells, HeLa B4GALTT ${ }^{-}$and $S L C 35 B 2^{-1-}$ cells showed particularly high resistance (>30-fold) against Tcna (Fig. 1d). Unlike EXT1, EXT2, and EXTL3, which only catalyze the heparan sulfate biosynthesis, B4GALT7 and SLC35B2 are involved in the biosynthesis of various sulfated glycans. B4GALT7 catalyzes the initiation of glycosaminoglycan side chain synthesis and SLC35B2 transports adenosine-3'-phospho-5'-phosphosulfate (PAPS), a sulfate donor, from the cytosol into the Golgi (Supplementary information, Fig. S1). To examine whether other sulfated glycosaminoglycans could also be recognized by Tcna, we performed competition assays using a panel of glycans structurally related to heparan sulfate. Pre-incubation of Tcna with heparan sulfate, chondroitin sulfate, heparin, dextran sulfate, and sulfated a-cyclodextrin (SCD) all reduced level of cell rounding, whereas hyaluronic acid, the non-sulfated glycosaminoglycan, had no inhibitory effect (Fig. 1f; Supplementary information, Fig. S3a). Consistently, pre-treatment of cells with surfen (bis-2-methyl-4amino-quinolyl-6-carbamide), a small molecule that neutralizes the negative charge of sulfated glycosaminoglycans, protected Hela cells from Tcna (Fig. 1g; Supplementary information, Fig. S3b). These results demonstrated that Tona recognizes sulfated glycans and the negatively charged sulfation group is critical for the interactions.

Synergistic actions between glycosaminoglycans and lowdensity lipoprotein receptor (LDLR) are common for cellular internalization of ligands. To demonstrate whether Tcna hijacks this pathway for toxin entry, we examined the sensitivity of HeLa $L D L R^{-/-}$cells to Tcna using the cell rounding assay. We also included the HeLa CSPG4 $4^{-1-}$ and $F Z D 1 / 2 / 7^{-1}$ cells, which are resistant to $\mathrm{TCdB}^{5}{ }^{5}$ for comparison. $L D L R^{-/-}$cells, but not $C S P G 4^{-/-}$ and $F Z D 1 / 2 / 7^{-1}$ cells, showed modestly reduced sensitivity ( 8fold) to Tcna (Fig. $1 \mathrm{~h}, \mathrm{i}$ ). On the other hand, surface binding of Tcna was diminished in $S L C 35 B 2^{-1-}$ cells but not $L D L R^{-/-}$and $\mathrm{CSPG}^{-1-}$ cells (Fig. $1 \mathrm{j}$ ), indicating that sulfated glycosaminoglycans but not LDLR mainly contribute to the surface attachment of Tcna. LDLR is rapidly and constitutively internalized and recycled between cell membranes and endosomes, and its cytosolic domain is critical for mediating these processes. ${ }^{11}$ We showed that the susceptibility of $L D L R^{-/-}$cells can be restored by transient transfection of full-length mouse Ldlr (Fig. 1k). Interestingly, transient transfection of an Ldlr mutant lacking the cytosolic domain (residues 1-824, Ldlr $\Delta \mathrm{C}$ ) also restored the susceptibility of $L D L R^{-/-}$cells but less efficiently (Fig. $1 \mathrm{k}, \mathrm{I}$ ), demonstrating that the fast-recycling ability of LDLR promotes the intoxication of Tcna in HeLa cells but is not essential. We could not detect obvious binding of Tcna to LDLR in vitro (Supplementary information, Fig. S4), indicating that either the interaction between Tcna and LDLR is weak or additional co-factors are needed. 
a

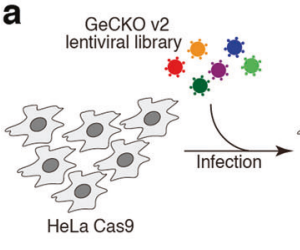

C

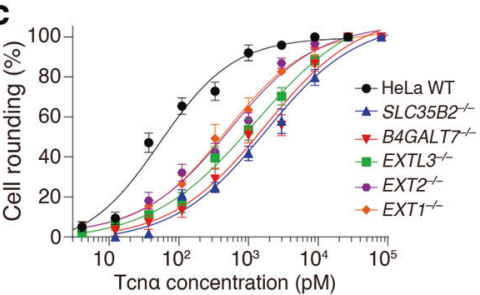

f

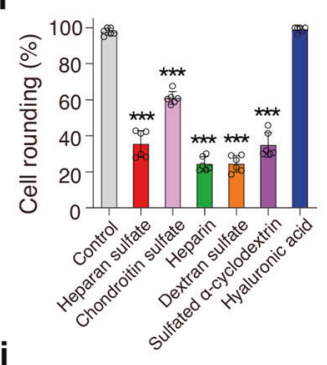

j
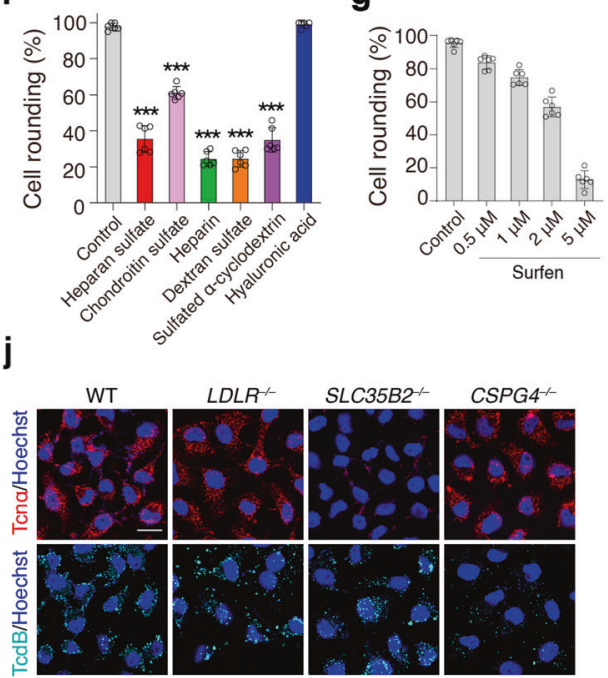

h
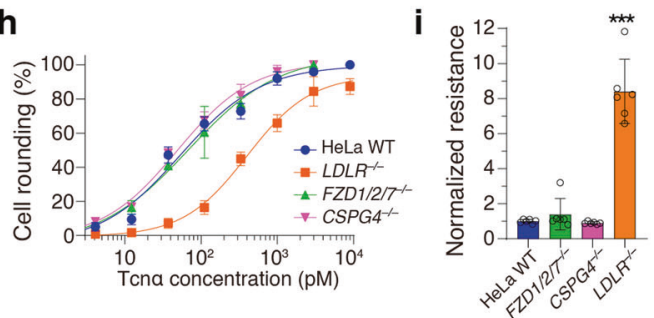

$\mathbf{k}$
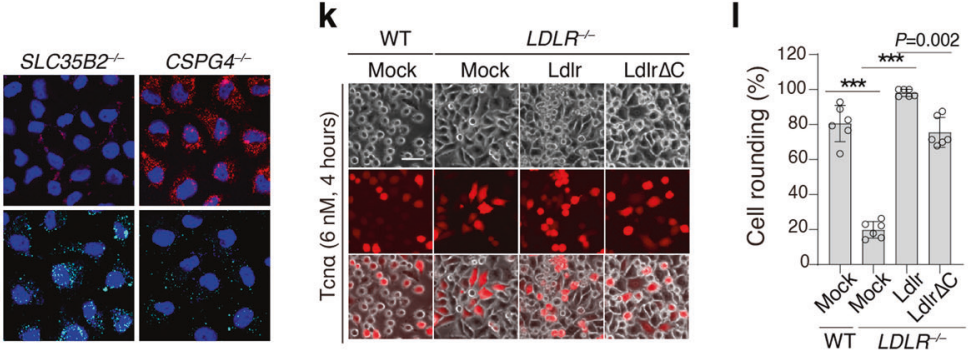

d
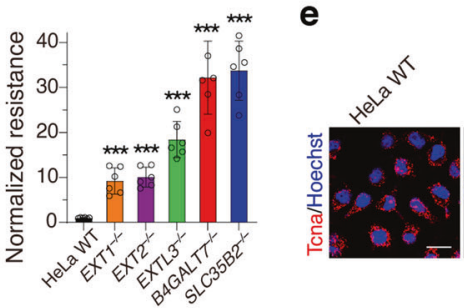

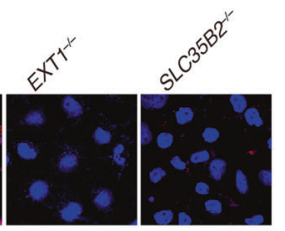

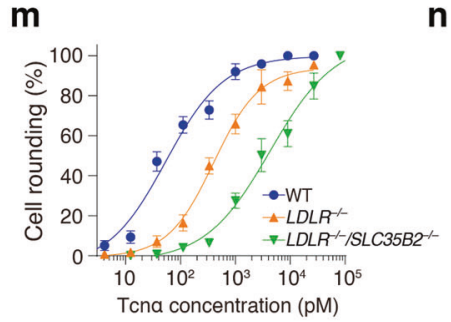

n

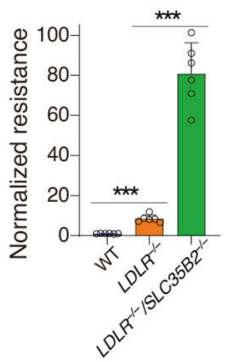

$\mathbf{q}$
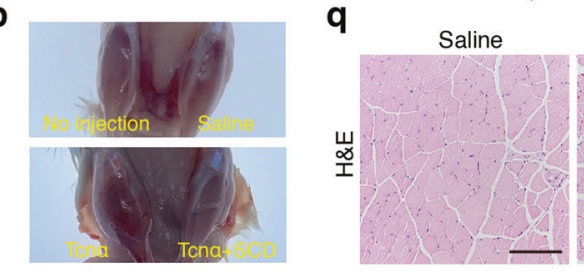

0
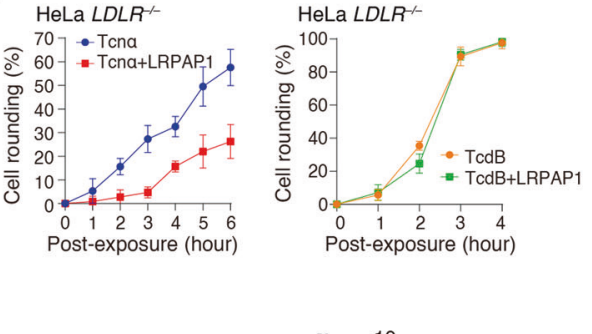

Tcna

Tcna+SCD

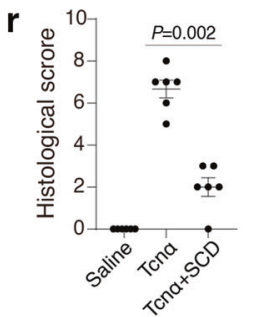

We next examined the sensitivity of the HeLa $L D L R^{-1-} / S L C 35 B 2^{-1-}$ cells to Tcna. These double KO cells showed further increased resistance to Tcna compared to the LDLR KO cells (Fig. $1 \mathrm{~m}, \mathrm{n}$ ), implying the existence of other endocytic portals besides LDLR. LDLR belongs to the LDLR family, of which members often act as redundant receptors for many ligands due to the similarity of their structural domains. Low-density lipoprotein receptor-related protein-associated protein 1 (LRPAP1) binds tightly to most LDLR family members and inhibits their ligand binding abilities. The addition of LRPAP1 at the molar ratio of 1:1000 (toxin vs LRPAP1) further reduced the sensitivity of $L D L R^{-/-}$cells to Tcna but not to TcdB (Fig. 10, Supplementary information, Fig. S5), indicating that 
Fig. 1 Sulfated glycosaminoglycans and LDLR mediate the cellular entry of Tcna. a Schematic of the screening process using Tcn $\alpha$ on HeLa cells with GeCKO v2 library. b Genes identified are ranked and plotted based on fold-enrichment of their gRNA from the beginning (R0) to 3round post-toxin selection (R3). Genes involved in heparan sulfate biosynthesis are marked. c Sensitivities of the WT, SLC35B2 ${ }^{-1-}$, B4GALT7 ${ }^{\prime-}$, EXTL3 ${ }^{-1-}, E X T 1^{-1-}$, and EXT2 ${ }^{-1-}$ HeLa cells to Tcn $\alpha$ were measured using the cytopathic cell-rounding assay. The percentages of rounded cells were quantified, plotted, and fitted. d Resistance of $S L C 35 B 2^{-/}, B 4 G A L T 7^{-1-}, E X T L 3^{-/-}, E X T 1^{-1-}$, and $E X T 2^{-/-}$HeLa cells to Tcn $\alpha$ were normalized to the level of WT cells based on $\mathrm{CR}_{50}$. ${ }^{* * *} P<0.001 \mathrm{vs}$ WT). e Confocal fluorescence images of Rhodamine-labeled Tcn $\alpha$ binding to the WT, EXT1 ${ }^{-1-}$, and SLC35B2 ${ }^{-/-}$HeLa cells. Cell nuclei were labeled with Hoechst. f HeLa cells were exposed to either Tcn $\alpha$ ( $\left.7.5 \mathrm{nM}\right)$ or Tcn $\alpha$ pre-mixed with the indicated glycans $(1 \mathrm{mg} / \mathrm{mL})$. The levels of cell rounding with $6 \mathrm{~h}$ incubation were plotted in a bar chart. (***P<0.001 vs control). $\mathbf{g}$ HeLa cells were pre-incubated with surfen of the indicated concentrations and then exposed to Tcn $\alpha(10 \mathrm{nM})$. The levels of cell rounding after $7 \mathrm{~h}$ incubation were plotted in a bar chart. $\mathbf{h}$ Sensitivities of the WT, $L D L R^{-1-}, F Z D 1 / 2 / 7^{-1}$, and CSPG4 ${ }^{-1-}$ HeLa cells to Tcn $\alpha$ were measured using the cytopathic cell-rounding assay. The percentages of rounded cells were quantified, plotted, and fitted. i Resistance of $L D L R^{-/-}, F Z D 1 / 2 / 7^{\prime-}$, and CSPG4 ${ }^{-1-}$ HeLa cells to Tcn $\alpha$ were normalized to the level of WT cells. (***P $<0.001$ vs WT). $\mathbf{j}$ Confocal fluorescence microscopy showing different binding of Rhodamine-labeled Tcn $\alpha$ or TcdB to the WT, $L D L R^{-1-}, S L C 35 B 2^{-1-}$, and CSPG4 ${ }^{-1-}$ HeLa cells. Cell nuclei were labeled with Hoechst. $\mathbf{k}$ Expression of mouse Ldlr restored Tcn $\alpha$ entry into HeLa $L D L R^{-/-}$cells, resulting in cell rounding; expression of mouse $L d l r \Delta C$ also restored Tcn $\alpha$ entry but less efficiently. Red fluorescence (mCherry) marked transfected cells. I Cell rounding among fluorescence positive cells shown in $\mathbf{k}$ was quantified and plotted in a bar chart. (***P $<0.001)$. $\mathbf{m}$ Sensitivities of the HeLa WT, $L D L R^{-1-}$, and $L D L R^{-1-} / S L C 35 B 2^{-1-}$ cells to Tcn $\alpha$ were measured using the cytopathic cell-rounding assay. The percentages of rounded cells were quantified, plotted, and fitted. $\mathbf{n}$ Resistance of $L D L R^{-1}$, and $L D L R^{-1-} / S L C 35 B 2^{-1-}$ HeLa cells to Tcn $\alpha$ were normalized to the level of WT cells. (***P<0.005). o LRPAP1 in culture medium further protected HeLa $L D L R^{-1-}$ cells from Tcn $\alpha$ (left panel, $5 \mathrm{nM}$ ) but not TcdB (right panel, $2.5 \mathrm{pM}$ ) under the molar ratio of 1:1000 (toxin vs LRPAP1). The percentages of rounded cells were quantified and plotted over time. $\mathbf{p}$ Anatomy of the mouse tibialis anterior muscles after i.m. injection of saline, Tcn $\alpha$, or Tcn $\alpha+$ SCD. q Representative images of H\&E staining sections from the mice tibialis anterior muscles after i.m. injection of saline, Tcn $\alpha$ alone, or $\mathrm{Tcn} \alpha+$ SCD. Asterisks indicate inflammatory cell infiltrate, solid arrowheads indicate hemorrhage, and hollow arrowheads indicate central nuclei. $\mathbf{r}$ Histopathological scores were accessed and summarized based on hemorrhage, inflammatory cell infiltration, and muscle fiber injury. Data are means $\pm S D, n=6$ in $\mathbf{c}, \mathbf{d}, \mathbf{f}-\mathbf{i}$, and I-o, two-sided Student's $t$-test. Data are means \pm SEM, $n=6$ in $\mathbf{r}$, Mann-Whitney test. Scale bar represents $50 \mu \mathrm{m}$ in $\mathbf{e}, \mathbf{j}$, and $\mathbf{k}$, and $100 \mu \mathrm{m}$ in $\mathbf{q}$.

other LDLR family members likely serve as redundant endocytic receptors for Tcna.

Recent studies showed that TcdA uses sulfated glycosaminoglycans as the cell surface attachment factors and LDLR family proteins as the entry receptors. ${ }^{8,12}$ The primary sequences between Tcna and TcdA are divergent, with only $~ 30 \%$ identity. In the LCT family, TcdA is most similar to TcsH ( $78 \%$ identity). We demonstrated that TcsH is equally toxic to the WT, $L D L R^{--}$, and $S L C 35 B 2^{-1-}$ cells (Supplementary information, Fig. S6a). We further tested the heparin-binding of major LCTs including TcdB, TcsL, $\mathrm{TcsH}$, and Tcna, only Tcna bound to the heparin-beads (Supplementary information, Fig. S6b).

Sulfated glycosaminoglycans are widely distributed in soft tissues including muscles, which are major pathologically relevant targets of Tcna. In vitro binding experiment showed that Rhodamine-labeled Tcna robustly bound to the tissue sections of mouse skeleton muscles. The toxin binding was drastically reduced when co-incubated with heparin or SCD, but not hyaluronic acid (Supplementary information, Fig. S7), suggesting that sulfated glycosaminoglycans are the major attachment factors mediating Tcna binding to the muscle fibroblast cells.

Finally, we investigated the role of sulfated glycosaminoglycans in Tcna-induced myopathogenesis in vivo using a toxin intramuscular (i.m.) injection model in mice. In brief, saline, Tcna, or Tcna premixed with SCD was injected into the mouse tibialis anterior muscles. Sulfated cyclodextrins had minimal cytotoxicity and hemolytic activity and are considered as potential ingredients in drug development and pharmaceutical applications. ${ }^{13}$ Therefore, we chose SCD as the inhibitor for the toxin protection assay in vivo. The tibialis anterior muscles injected with Tcna were visually swollen and congested (Fig. 1p). Hematoxylin and eosin $(\mathrm{H} \& \mathrm{E})$ staining showed that i.m. injection of Tcna resulted in severe hemorrhage and inflammatory cell infiltration. Central nuclei, the morphological marker for myopathies such as early necrosis, were also observed (Fig. 1q, r; Supplementary information, Fig. S8). The muscles co-injected with Tcna and SCD were less swollen and congested than those injected with Tcna alone (Fig. 1p). Histological analysis further revealed that co-injection of SCD drastically reduced Tcna-induced hemorrhage, inflammatory cell infiltration, and central nuclei (Fig. 1q, r; Supplementary information, Fig. S8).
In summary, we identified sulfated glycosaminoglycans as key attachment factors for Tcna via CRISPR/Cas9-mediated KO screens. Sulfated glycosaminoglycans are ubiquitously present on the cell surface thus serve as ideal landing pads for various infectious agents including SARS-COV-2, the emerging virus that causes global pandemics. ${ }^{14,15}$ We further revealed that the synergistic actions between sulfated glycosaminoglycans and LDLR family members facilitate the entry of Tcna. Finally, we demonstrated that sulfated glycosaminoglycans are key attachment factors responsible for Tcna-induced myopathies in vivo, and SCD effectively reduced the tissue damage caused by Tona in the mouse model. These findings suggest that blocking the interactions between Tcna and sulfated glycosaminoglycans could be a novel strategy for developing potential therapeutics against Tcnainduced diseases.

\section{ACKNOWLEDGEMENTS}

We are grateful to the Biomedical Research Core Facilities and Laboratory Animal Resources Center of Westlake University for providing technical support. This study was partially supported by the National Natural Science Foundation of China (31970129 and 31800128 to L.T.) and Zhejiang Provincial Natural Science Foundation of China (LR20C010001 to L.T. and LR20C050001 to Y.L.). L.T. also acknowledges support from Westlake Education Foundation and Tencent Foundation.

\section{AUTHOR CONTRIBUTIONS}

Y.Z. and L.T. conceived the project and designed the experiments. Y.Z. performed the CRISPR screens. Y.Z. and Diyin L. conducted most of the cell biology and biochemistry experiments. Y.Z and J.L. performed the mouse i.m. injection assay and histology analysis. A.C., X.L., and L.H. helped with the cell biology experiments and data analysis. Z.P., Danyang L., L.W., and Y.L. purified the proteins and labeled the toxin. M. D. provides key reagents and suggestions. L.T. wrote the manuscript with input from all co-authors.

\section{ADDITIONAL INFORMATION}

Supplementary information The online version contains supplementary material available at https://doi.org/10.1038/s41422-021-00510-z.

Competing interests: The authors declare no competing interests. 
Yao Zhou ${ }^{1,2,3}$, Diyin $\mathrm{Li}^{1,2,3}$, Jianhua Luo ${ }^{1,2,3}$, Aizhong Chen ${ }^{1,2,3}$ Xingxing $\mathrm{Li}^{1,2,3}$, Zhenrui Pan ${ }^{1,2,3}$, Li Wan ${ }^{1,2,3}$, Liuqing He $\mathrm{He}^{1,2,3^{\prime}}$ Danyang $\mathrm{Li}^{1,2,3}$, Yanyan $\mathrm{Li}^{1,2,3}$, Min Dong $\mathbb{i D}^{4,5}$ and Liang Tao (iD) ${ }^{1,2,3}$ ${ }^{1}$ Key Laboratory of Structural Biology of Zhejiang Province, School of Life Sciences, Westlake University, Hangzhou, Zhejiang, China; ${ }^{2}$ Center for Infectious Disease Research, Westlake Laboratory of Life Sciences and Biomedicine, Hangzhou, Zhejiang, China; ${ }^{3}$ Institute of Basic Medical Sciences, Westlake Institute for Advanced Study, Hangzhou, Zhejiang, China; ${ }^{4}$ Department of Urology, Boston Children's Hospital, Boston, MA, USA and ${ }^{5}$ Department of Surgery and Department of Microbiology, Harvard Medical School, Boston, MA, USA

These authors contributed equally: Yao Zhou, Diyin Li, Jianhua Luo Correspondence: Liang Tao (taoliang@westlake.edu.cn)

\section{REFERENCES}

1. Hatheway, C. L. Clin. Microbiol. Rev. 3, 66-98 (1990).

2. Aronoff, D. M. \& Kazanjian, P. H. Anaerobe 50, 80-84 (2018).

3. Jank, T. \& Aktories, K. Trends Microbiol. 16, 222-229 (2008).

4. Schorch, B. et al. Proc. Natl. Acad. Sci. USA 111, 6431-6436 (2014).

5. Tao, L. et al. Nature 538, 350-355 (2016).

6. Yuan, P. et al. Cell Res. 25, 157-168 (2015).

7. LaFrance, M. E. et al. Proc. Natl. Acad. Sci. USA 112, 7073-7078 (2015).
8. Tao, L. et al. Nat. Microbiol. 4, 1760-1769 (2019).

9. Lee, H. et al. Cell 182, 345-356 (2020).

10. Tian, S. et al. Cell Host Microbe 27, 782-792 (2020).

11. Chen, W. J., Goldstein, J. L. \& Brown, M. S. J. Biol. Chem. 265, 3116-3123 (1990).

12. Schottelndreier, D. et al. Front Cell. Infect. Microbiol. 10, 565465 (2020).

13. Roka, E. et al. Molecules 20, 20269-20285 (2015).

14. Clausen, T. M. et al. Cell 183, 1043-1057 (2020).

15. Zhang, Q. et al. Cell Discov. 6, 80 (2020).

(i) Open Access This article is licensed under a Creative Commons Attribution 4.0 International License, which permits use, sharing, adaptation, distribution and reproduction in any medium or format, as long as you give appropriate credit to the original author(s) and the source, provide a link to the Creative Commons license, and indicate if changes were made. The images or other third party material in this article are included in the article's Creative Commons license, unless indicated otherwise in a credit line to the material. If material is not included in the article's Creative Commons license and your intended use is not permitted by statutory regulation or exceeds the permitted use, you will need to obtain permission directly from the copyright holder. To view a copy of this license, visit http://creativecommons. org/licenses/by/4.0/.

(c) The Author(s) 2021 